\title{
Comparison of regional gene expression differences in the brains of the domestic dog and human
}

\author{
Erin Kennerly,' Susanne Thomson, ${ }^{2}$ Natasha Olby, ${ }^{3}$ Matthew Breen ${ }^{2}$ and Greg Gibson ${ }^{1 *}$ \\ 'Department of Genetics, College of Agriculture and Life Sciences, North Carolina State University, Raleigh, NC 27695, USA \\ ${ }^{2}$ Department of Molecular Biomedical Sciences, College of Veterinary Medicine, North Carolina State University, Raleigh, \\ NC 27606, USA \\ ${ }^{3}$ Department of Clinical Sciences, College of Veterinary Medicine, North Carolina State University, Raleigh, NC 27606, USA \\ *Correspondence to: Tel: +1 919513 2512; Fax: +1 919515 3355; E-mail: ggibson@unity.ncsu.edu
}

Date received (in revised form): 22nd October 2004

\begin{abstract}
Comparison of the expression profiles of 2,72I genes in the cerebellum, cortex and pituitary gland of three American Staffordshire terriers, one beagle and one fox hound revealed regional expression differences in the brain but failed to reveal marked differences among breeds, or even individual dogs. Approximately 85 per cent (42 of 49 orthologue comparisons) of the regional differences in the dog are similar to those that differentiate the analogous human brain regions. A smaller percentage of human differences were replicated in the dog, particularly in the cortex, which may generally be evolving more rapidly than other brain regions in mammals. This study lays the foundation for detailed analysis of the population structure of transcriptional variation as it relates to cognitive and neurological phenotypes in the domestic dog.
\end{abstract}

Keywords: canine, breed, microarray, cerebellum, cortex, pituitary

\section{Introduction}

Gene expression profiling provides a novel perspective from which to consider the degree of genetic differentiation of individuals within populations. The domestic dog, Canis familiaris, is an excellent organism for this pursuit, since phenotypic and nucleotide divergence are not highly correlated. Whereas breeds are clearly, and often discretely, differentiated morphologically and behaviourally, resolution of genetic relatedness among breeds requires a large number of anonymous microsatellite markers. ${ }^{1-4}$

The question thus arises as to whether divergence at the gene expression level is greater within or among breeds. There is a clear expectation that some fraction of the transcriptome in particular tissues and at appropriate phases of development will correlate with phenotypic variation. Similarly, disease status ought to reflect transcriptional changes, 5,6 but any such inference must be assessed against a background knowledge of the degree of standing transcriptional variation. $^{7}$

Quantitative comparison of transcriptomes requires statistically orientated analytical methods that can partition the effects of multiple sources of variance. We and others have introduced linear analysis of variance algorithms for microarray data, ${ }^{8,9}$ and Bayesian procedures have been employed that perform similarly. ${ }^{10,11}$ With appropriate experimental design and moderate replication, it is straightforward to demonstrate that changes in expression smaller than twofold are significant experiment-wide. Furthermore, these approaches take into account variance contributions from each factor when assessing specific effects and powerfully demonstrate interaction effects. For example, in a study of the influence of sex, age and genotype on gene expression in Drosophila, we showed that a substantial fraction of the transcriptome differs more between genotypes for just one of the sexes, while age has only a very modest effect on transcription. $^{12}$

The objective of this study was to begin to assess the extent to which gene expression differs within and among breeds of dog in three parts of the brain. For many species, it has been shown that between 5 and 20 per cent of genes are differentially expressed between individuals, and the mammalian brain is no different. ${ }^{13,14}$ The left prefrontal lobe (Brodmann area 9) of three human brains differs from the 
homologous region of the chimpanzee brain at over 1,000 loci, although, remarkably, one human was found to differ from the others by at least as much as all three differ from three chimpanzees. ${ }^{15,16}$ Follow-up comparison of several regions of the human brains suggested that there is more variation among individuals than between parts of the cortex, although it is not clear whether this is due to genetic or environmental factors. ${ }^{17}$ Similarly, an earlier study comparing normal and postseizure mouse brains highlighted strain differences among brain regions. ${ }^{18,19}$ Recently, human Affymetrix chips were used to detect some divergence in transcript abundance between pools of brain tissue from several domestic dogs and two wild canid species. ${ }^{20}$ Here, we conducted a complementary experiment, employing a canine brain cDNA microarray to contrast region-specific expression in five individual domestic dog brains. We discuss the nature of the genes that differentiate the cortex, cerebellum and pituitary gland of C. familiaris, and argue that differences among individuals are likely to be more prevalent than are breed-specific differences.

\section{Methods}

\section{Microrarrays}

A canine brain expressed sequence tag (EST) library consisting of approximately 4,600 unique ESTs, most of which have partial sequence and preliminary annotation, was obtained from Dr James Mickelson at the University of Minnesota. ${ }^{21}$ Construction of our 4,224 spot cDNA microarray is described elsewhere (Thomson et al., paper submitted). Tentative annotation of many of the ESTs using BLAST matches to end sequence and GenBank accession numbers is provided online as supplementary Table 1 at http://statgen.ncsu.edu/ ggibson/SupplInfo/SupplInfo9.htm, along with the raw fluorescence intensities and MIAME-compliant description of the experiment. Note that a comprehensive Affymetrix short oligonucleotide canine microarray has also just been described, ${ }^{22,23}$ as have two small targeted cardiovascular arrays. $^{24,25}$

Dissected brains from five adult dogs that were presented to the North Carolina State University Veterinary Teaching Hospital were used as the source of mRNA from pituitary gland, cortex and cerebellum. All dogs were euthanised and subjected to necropsy at the request of the owners for medical reasons. The dogs included three American Staffordshire terriers, one beagle and one American foxhound. The brains were removed in a sterile fashion within 30 minutes of death, the meninges were dissected away and tissues were taken from the frontal cortex, lateral cerebellar hemispheres and pituitary gland. These were snap frozen in liquid nitrogen and stored at $-80^{\circ} \mathrm{C}$. RNA isolation was performed after addition of $1 \mathrm{ml}$ per $50-100 \mathrm{mg}$ tissue of TRIzol reagent (Invitrogen) following the manufacturer's

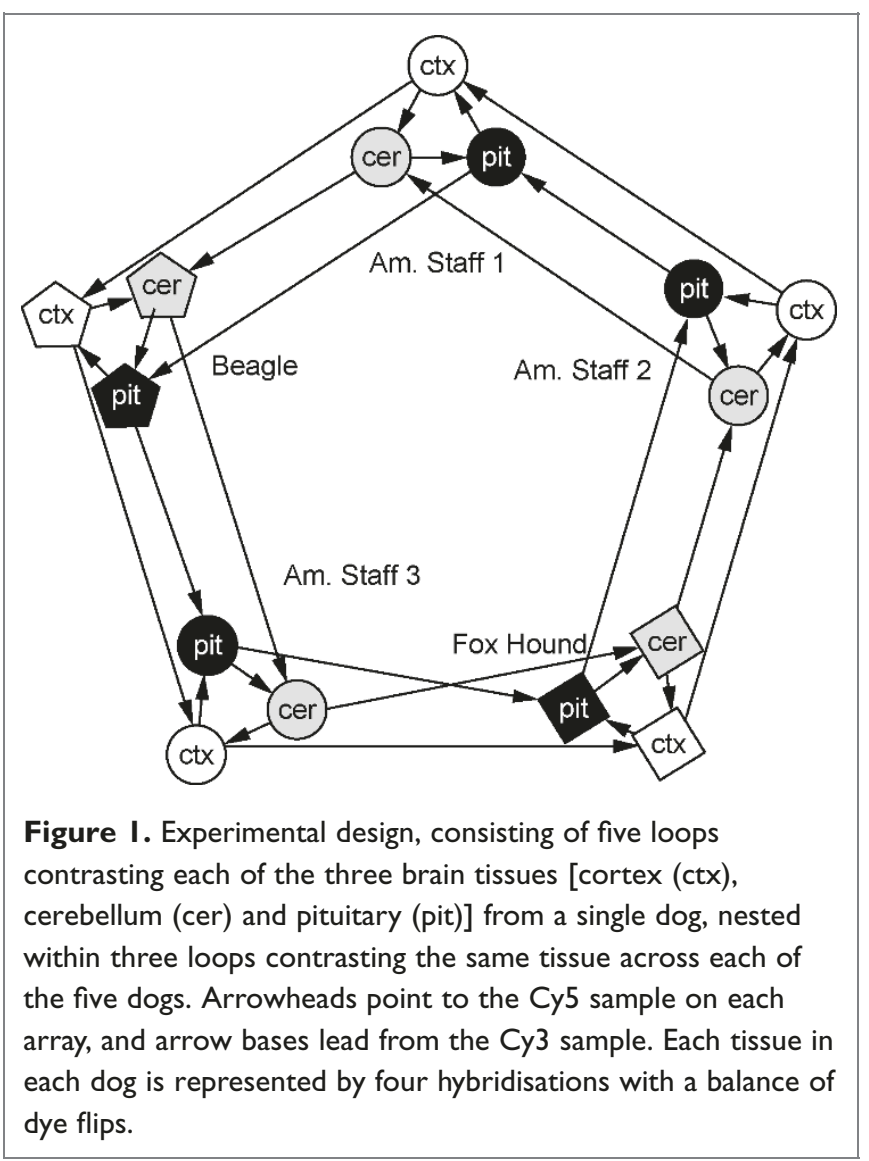

instructions, with further purification using an Rneasy Mini Kit (Qiagen). The quality and purity of RNA was analysed on a 0.8 per cent agarose gel and by taking $260 \mathrm{~nm} / 280 \mathrm{~nm}$ absorbance readings on a spectrophotometer.

The experimental design shown in Figure 1 consists of one main loop, where each tissue type was compared across different breeds, and five smaller loops in which each tissue within a single dog was compared with other tissues in that same dog. Linear RNA amplification ${ }^{26}$ was performed using an Agilent Low RNA Input Fluorescent Linear Amplification Kit (Product number 5184-3523). A single round of amplification was performed using $500 \mathrm{ng}$ of total RNA, yielding up to $50 \mu \mathrm{g}$ of amplified complementary RNA (acRNA). Occasionally, a second amplification reaction was needed to obtain enough acRNA. When this occurred, the two reactions were pooled. First-strand cDNA was synthesised from $3 \mu \mathrm{g}$ of acRNA using Improm II reverse transcriptase (Promega) in four separate reactions for each sample. After purification of first-strand cDNA, the four reactions were pooled and re-split to reduce variations between individual cDNA synthesis reactions. Amplified cDNA was then labelled indirectly through an aminoallyl linkage with Cy3 or Cy5 in a balanced manner (using The Institute for Genomic Research protocol SOP\#M004), 
resulting in two $\mathrm{Cy} 3$ and two $\mathrm{Cy} 5$ reactions per sample. Hybridisations were performed for 20 hours at $42^{\circ} \mathrm{C}$, followed by washing in a standard series of highstringency washes. Microarray slides were scanned using a ScanArray 4000 Microarray Analysis System Scanner (Packard Bioscience). ScanAlyze 2 (http://rana.lbl.gov/ EisenSoftware.htm) ${ }^{27}$ was used to generate data files from the acquired images.

\section{Data analysis}

Raw fluorescence intensities from Scanalyze 2 were further analysed using a two-step mixed model analysis of variance procedure $^{8,28}$ in SAS Version 5.0 (SAS Institute, Cary, NC). Raw fluorescence intensities were log transformed on the base 2 scale, and the 1,503 spots with the lowest average expression across all arrays were removed from consideration. This number was selected because they lay below the inflection point of a plot of rank-ordered average raw fluorescence intensity for all of the spots on the array. Spots at or below this point (raw values $186 ; \log _{2}$ value 7.54 ) are no more intense than the mean background intensity level across all array. All of the remaining 2,721 spots were then normalised with a first analysis of variance model that adjusts for overall array and dye effects. Residuals from this model are relative fluorescence intensities ( $\left.\log _{2} \mathrm{RFI}\right)$ for each gene, essentially a measure of the fold difference in expression level for each gene relative to the sample mean for the appropriate channel on each array.

These intensities were then compared on a gene by gene basis, accounting for the variance among dogs and tissues according to gene-specific mixed models of the form:

$$
\log _{2} \mathrm{RFI}_{i j k l}=\mu+C_{i}+T_{j}+D_{k}+C \times T_{i j}+A_{l}+\varepsilon_{i j k l}
$$

where fixed effects are represented by $C$ for the $i$ th individual canine $(i=1, \ldots, 5), T$ for the $j$ th tissue (cerebellum, cortex, or pituitary) and $D$ for the kth dye (Cy3 or Cy5). The term $C \times T_{i j}$ fits the interaction between $\operatorname{dog}$ and tissue, while the random effects of the $l$ th array $(l=1, \ldots, 30)$ are presumed to be normally distributed with mean zero and variance $\sigma^{2}$. The mean and unexplained error are represented by $\mu$ and $\varepsilon$, respectively. This procedure obviates the need for a reference sample and assesses the significance of gene expression differences between samples relative to the variance in measurements of each sample type. The online Results Supplementary Table 2 shows the significance of the $C, T, C \times T$ and $D$ terms (columns $\mathrm{C}$ to $\mathrm{F}$ ), along with the amount of variance explained by each gene-specific model (column B). Subsequently, the magnitude and significance of the difference in expression of the three American Staffordshire terriers from the single foxhound or beagle individual, and of the three brain regions (cortex, cerebellum and pituitary) were computed using the DIFFS option in PROC MIXED, using SAS code that is available on request. As described in the text, the significance threshold of $p<0.0001$ was adopted for gene selection, as none of the 2,721 genes in the analysis are expected to be significant at this level by chance. Clustering in Figure 2 was performed according to Ward's method on the standardised means of the four measurements from each dog, using JMP Version 5.0 software (SAS Institute, Cary, NC, USA). The genes listed in Table 1 meet the more stringent Bonferroni cutoff $(p<0.00002)$.

Comparison with the Novartis Human Gene Expression Atlas $^{29}$ was performed using the online text query feature at http://expression.gnf.org/cgi-bin/index.cgi. This resource provides the results of duplicate (cortex and cerebellum) or single (pituitary gland) human tissue hybridisations performed against the Affymetrix Human U95A platform. Since pituitary is not represented in similar mouse data, our dog results were only compared with human. Genes listed in Table 1 that were significantly differentially regulated in the dog were individually queried. Since no statistical measures are provided online, genes whose expression was twice as high (or twice as low) in the indicated tissue relative to the other two tissues, in both species, were regarded as being consistently regulated.

For the reciprocal comparison of differentially regulated human genes, we first used the online filter to identify sets of genes in the cortex, cerebellum and pituitary that are below the average, or more than twice the average, of the 46 Novartis tissues. Pairwise comparison of these lists identifies a subset of all genes that are at least twofold differentially regulated between the tissues, which numbers between 175 and 453 genes depending on the comparison. The annotations of these genes and the canine gene accessions were then scanned for exact matches. Owing to the relatively small sample of canine genes and incomplete annotation, only around 5 per cent of the human genes could be matched to canine genes. In these cases, we asked whether the difference in expression on our arrays was significantly different in the same direction (replicated), in the same direction but not significantly so (consistent), not differentially expressed (questionable) or significant in the other direction to that seen in humans (opposite effect). Human pituitary-specific genes are apparently under-represented on our canine array, so only three clones could be compared, all of which were also upregulated in the pituitary of the dog.

\section{Results}

Differential expression between brain regions Of the 4,224 genes represented on our microarray, 2,721 were expressed above background levels in at least one tissue, with 591 genes showing nominal testwise significant differences $(p<0.05)$ in transcript abundance between the three brain regions. By contrast, at this 5 per cent significance level, just 
Table I. List of differentially expressed genes by brain region.

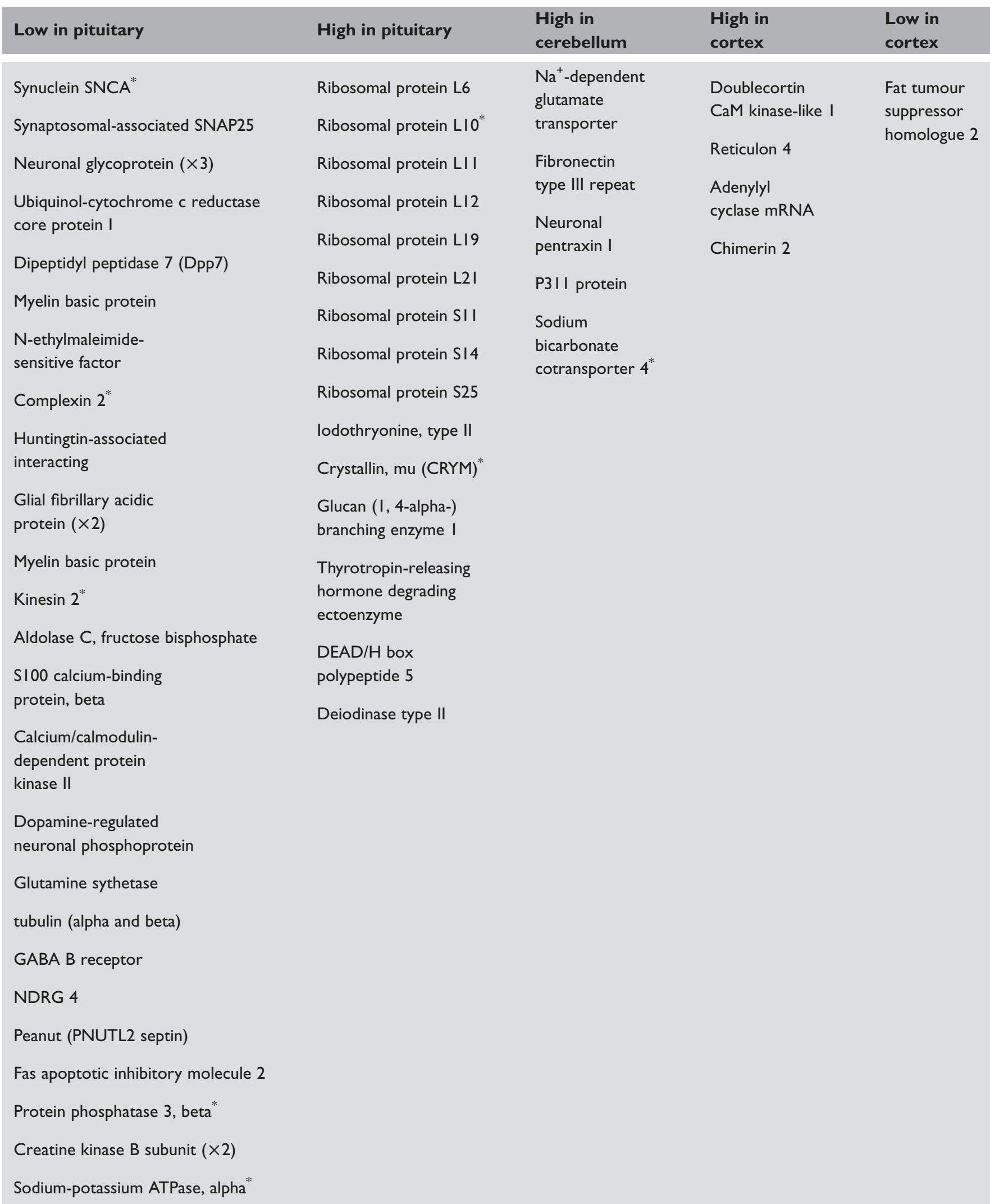

* Genes that do not show the same expression bias in the Novartis Human Gene Expression Atlas (see Supplementary Information). 
Table 2. Number of differentially expressed genes by brain region.

\begin{tabular}{|lllllrr|}
\hline Significance level & $\begin{array}{l}\text { High in } \\
\text { pituitary }\end{array}$ & $\begin{array}{l}\text { High in } \\
\text { cereb }\end{array}$ & $\begin{array}{l}\text { High in } \\
\text { cortex }\end{array}$ & $\begin{array}{l}\text { Low in } \\
\text { pituitary }\end{array}$ & $\begin{array}{l}\text { Low in } \\
\text { cortex }\end{array}$ & 142 \\
\hline$p<0.00002$ (Bonferroni) & 30 & 13 & 20 & 95 & 3 & 11 \\
\hline$p<0.000$ I (FDR) & 73 & 49 & 22 & 301 & 274 & 236
\end{tabular}

Abbreviations: Cereb, cerebellum; FDR, false discovery rate.

139 genes differed among the five dogs, and 131 genes differed among the dogs in a region-specific manner, which is precisely the number of genes expected by chance. Consequently, the experiment provided good evidence for the differential expression of up to 15 per cent of the genes among brain regions but no strong evidence for differential expression between dogs.

The numbers of genes differentially expressed at significantly higher levels in one of the three brain tissues than in both of the other two are indicated in Table 2. At the significance cutoff of $p<0.0001$, no significant differences are expected by chance, so the false discovery rate (FDR) is minimised. A total of 290 expression differences was observed, however: expression was elevated for 73 genes in the pituitary, 49 in the cerebellum and 22 in the cortex, while 135 genes were noticeably repressed in the pituitary and 11 genes showed their lowest expression in the cortex. Since no genes were lower in the cerebellum than in the cortex and pituitary there are thus five clusters of differentially expressed genes that appear in the two-way hierarchical cluster heat map in Figure 2. Table 2 further indicates that differential expression trends were also seen at more stringent (Bonferroni) or less stringent (testwise) significance cutoffs, confirming that gene expression was most divergent in the pituitary. The identities of the annotated genes in each class are listed in Table 1 and are discussed below.

\section{Relative absence of differentiation among dogs}

Two further tests for differentiation between animals and breeds failed to provide any formal evidence for global differentiation among dogs. Figure 3 presents volcano plots of significance against magnitude of expression difference for each of the three pairwise contrasts of American Staffordshire terrier (three dogs) against foxhound and beagle (one dog each). Significance on the Y-axis is plotted as the negative logarithm of the $p$-value, such that values exceeding the $p=0.0001$ threshold are above the dotted horizontal line.

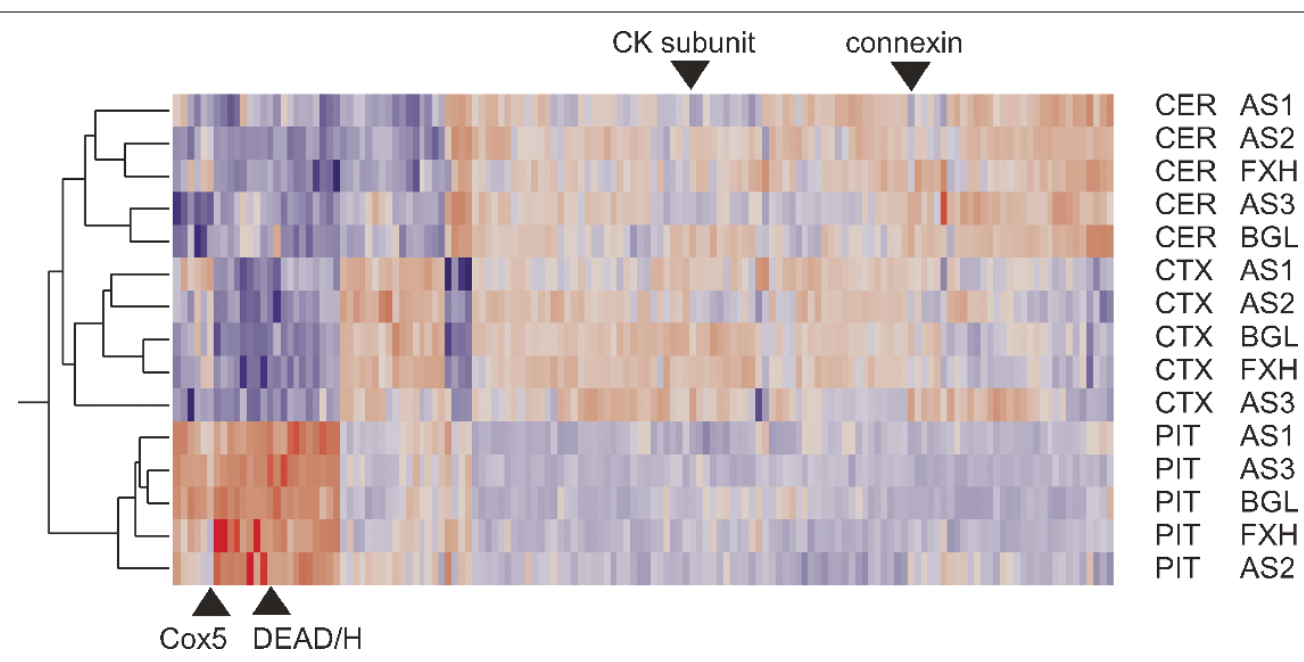

Figure 2. Heat map showing two-way hierarchical clustering of standardised least-square means of transcript abundance over the four hybridisations. Each row represents the indicated brain region from one dog. Each column represents one gene for which significant expression differences were observed, either among brain regions or breeds, at $p<0.000 \mathrm{I}$. Red indicates relatively high expression, blue low expression. Triangles highlight genes mentioned in the Discussion showing bimodal abundance within the pituitary or cerebellum. 


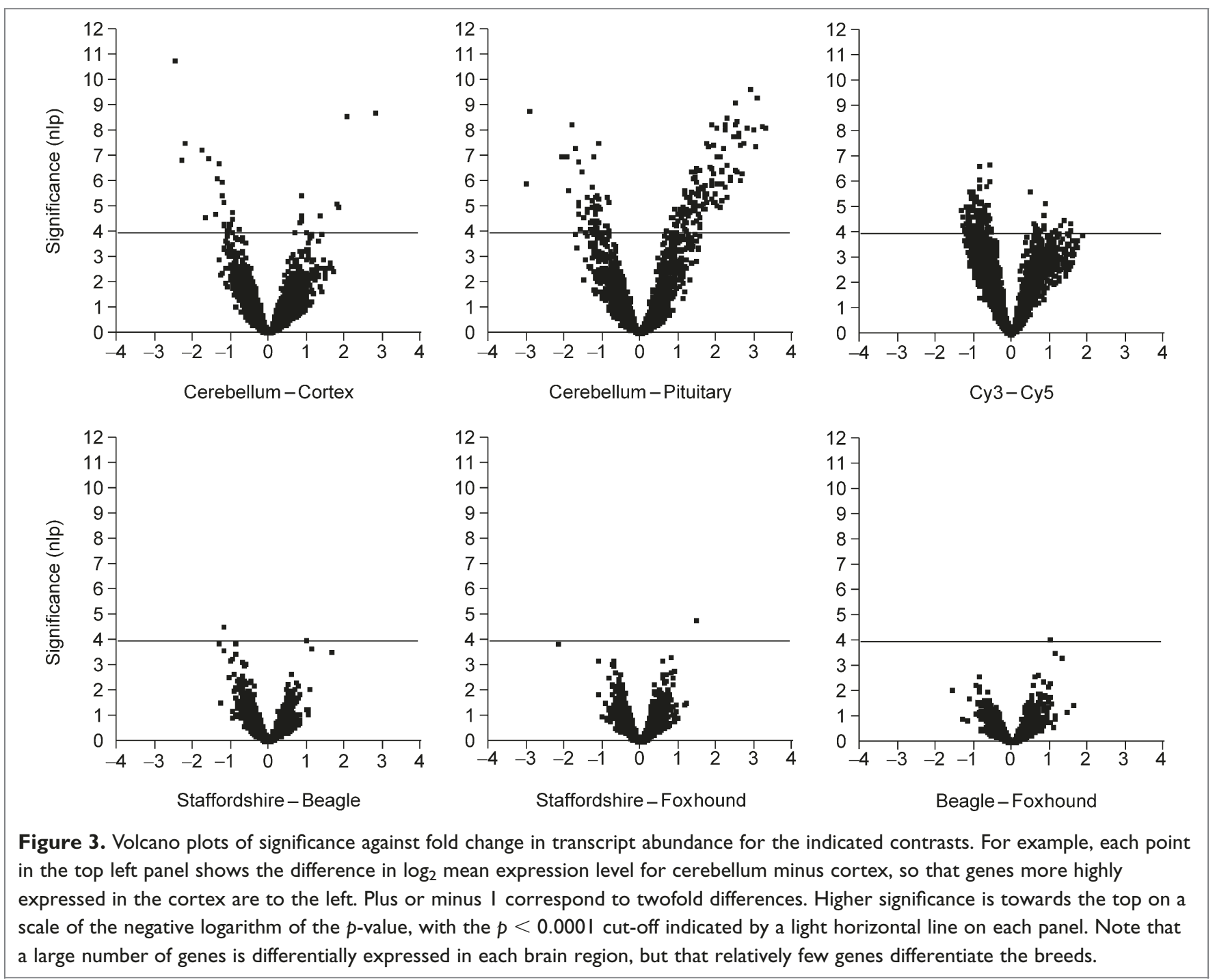

Fold change in transcript abundance is shown on the log base 2 scale along the $\mathrm{X}$-axis. Only a handful of genes appeared to be differentially expressed across the three brain regions between each pair of breeds.

Similarly, the clustering of dogs in Figure 2 tends to indicate that any differential gene expression between dogs within brain regions also is not breed specific. Transcript abundance is remarkably uniform in the five pituitaries, while between 20 and 30 transcripts differentiate each dog from each other dog in the cerebellum. In the cortex, one of the American Staffordshire terriers is quite different from the other four dogs and there is a suggestion that the beagle and foxhound are more similar to one another than to the American Staffordshire terriers. Even though the same clustering pattern is observed when different numbers of genes are included in the analysis, it is due to just a handful of genes. Greater sampling depth and/or replication would undoubtedly elevate several percent of the genes represented in the transcriptome to the status of formally significant differential expression between individual dogs, but very few of these differences are likely to be breed specific.

\section{Discussion}

Transcriptional divergence between the pituitary and the cortex and the cerebellum generally reflects the hormonal and neuronal roles of these regions of the brain. Notable among the genes with relatively low expression in the pituitary are synaptic proteins, neuronal glycoproteins and several that encode proteins and enzymes related to neurotransmitter activity. By contrast, genes upregulated in the pituitary include a thyrotropin-releasing hormone degrading enzyme, iodothyronine and multiple ribosomal proteins, consistent with the notion that the pituitary is a site of 
Table 3. Comparison of differential expression in human and $\operatorname{dog}^{\mathrm{a}}$

\begin{tabular}{|c|c|c|c|c|c|}
\hline Comparison & Total $^{\text {b }}$ & Replicated & Consistent & Questionable & Opposite \\
\hline Cerebellum $>$ pituitary & 403 & 8 & 7 & 5 & I \\
\hline Cortex $>$ pituitary & 453 & 8 & 4 & 9 & 3 \\
\hline Cerebellum $>$ cortex & 175 & 3 & 3 & 1 & 0 \\
\hline Cortex $>$ cerebellum & 286 & 0 & 4 & 4 & I \\
\hline
\end{tabular}

${ }^{a}$ Genes differentially expressed in humans showing the same direction of effect in dogs that is significant at $p<0.05$ (replicated), non-significant (consistent), no change or non-significant in the opposite direction (questionable), or significant in the opposite direction.

${ }^{b}$ The total number of Affymetrix probe-sets reported as being more than two-fold higher in the first tissue than the mean of all other tissues, and less than the mean of all others in the second tissue, from the Novartis website. ${ }^{29}$ Only around 5 per cent of these probe-sets have unambiguous orthologues in this canine cDNA array. Too few pituitary-specific human genes were detected on the canine array to report a contrast.

enhanced protein synthesis. Differentiation of the cortex and cerebellum is less pronounced, but includes genes with clear neuronal functions such as a glutamate transporter, fibronectin repeat protein and pentraxin, which are upregulated in the cerebellum, and an adenylyl cyclase and calmodulindependent protein kinase which are upregulated in the cortex.

\section{Comparison of human and canine region- specific gene expression in the brain}

Comparison with online data from the Novartis Gene Expression Atlas ${ }^{29}$ indicates that around 85 per cent

(42 out of 49) of our annotated dog genes that are orthologous to unique human genes show similar differences among brain regions. This indicates that much of the functional differentiation between the cerebellum, cortex and pituitary at the gene expression level has been retained over tens of millions of years, irrespective of differences in brain size. Among the genes highlighted with asterisks in Table 1 that do not show consistent profiles across the two species, most are members of gene families, suggesting either that precise annotation of the short dog EST sequences is misleading or that subfunctionalisation among paralogous genes occurs at a reasonable frequency. ${ }^{30}$

A reciprocal analysis, namely ascertainment of which differentially expressed genes from the Novartis Human Data Index are also differentially expressed in dogs, suggests that the cortex may be more different than the cerebellum between these species. As summarised in Table 3, over two-thirds of the 21 genes upregulated in the cerebellum relative to the pituitary that have orthologues on both sets of microarrays are also upregulated in the dog, while just one is significantly higher in the canine pituitary. By contrast, just half of the 24 orthologous genes upregulated in human cortex relative to cerebellum are also upregulated in dogs, and three genes are significantly differentially transcribed in the opposite direction. Furthermore, five of nine genes upregulated in the human cortex relative to the cerebellum are not upregulated in the dog, whereas six of seven genes more highly expressed in the human cerebellum than the cortex show the same pattern in the dog. Contrasting humans with chimpanzees similarly suggests more extensive divergence of expression in the cortex than in three other brain regions. ${ }^{17}$ More intensive profiling, combined with molecular evolutionary analysis of sequence divergence, is a promising strategy for discovery of genes that may contribute to cognitive evolution and neuropathology. ${ }^{31}$

\section{Expression variation in dogs and wild canids}

As noted, no formally significant differences in gene expression between the dogs or breeds were detected. This is a little surprising, given that similar-sized studies in flies, ${ }^{12,32,33}$ fish, ${ }^{34}$ mice $^{19}$ and humans ${ }^{13,15}$ have all found evidence for differences of approximately 10 per cent of the transcriptome between individuals. A recent comparison of pools of mRNA from three Labrador retrievers and seven German shepherds with pools from ten coyotes or five grey wolves ${ }^{20}$ detected differential expression involving at least 114 genes between all three species in the amygdala and frontal lobe or between dogs and wild canids in the hypothalamus. Four of these genes were retested by quantitative reverse transcriptase polymerase chain reaction in samples from individual animals, and while two- to fourfold differentiation between species was confirmed, no differences between individual dogs were detected. Power computations indicate that detection of differential expression at levels less than 1.5-fold would, given the technical variance in our cDNA arrays, generally require more than the four replicates reported here. The trend detected in this study is that transcript abundance tends to be uniform among dogs and, as far as the very limited sample is concerned, across breeds of dogs. Nevertheless, it is likely that a broader survey encompassing different stages of brain development, or a larger sample of dogs with more replication, would detect genes whose expression varies among individuals either for genetic or environmental reasons.

It is well known from human genetics that behaviourallyrelated loci, such as monoamine oxidase and the serotonin transporter, are expressed at different levels among 
individuals. ${ }^{35,36}$ These genes are not represented on our array, so it is not yet clear whether expression is polymorphic in dogs as well. Close inspection of Figure 2 reveals several dozen genes whose expression is greater in two or three of the dogs than in the others and post hoc tests suggest these as candidate genes for differential regulation across individuals. Examples indicated on Figure 2 include cytochrome c oxidase subunit COX5B (our clone identity number DG1314) and a $\mathrm{DEAD} / \mathrm{H}$ box protein (DG0610) in the pituitary, and a creatine kinase subunit (DG3263) and complexin (DG3512) in the cerebellum. Most of these cases show sharing of the two transcriptional states across breeds, implying that any efforts to correlate gene expression with behavioural divergence in dogs should be conducted across a broad range of breeds to avoid the effect of population stratification on inference of genetic association.

\section{Acknowledgments}

We thank Jim Mickelson for providing the sequenced cDNA clones, the owners of the dogs that provided tissues for this study and Kevin Woollard for assistance with the craniotomies. This research was supported by NIH award R01 GM61600 to GG, and by awards from the American Kennel Club Canine Health Foundation as well as the NCSU College of Veterinary Medicine to $\mathrm{MB}$.

\section{Electronic database information}

The complete list of genes on our microarray, raw fluorescence intensities, as well as the results of the Mixed Model Analysis of Variance are available online at: http://statgen. ncsu.edu/ggibson/SupplInfo/SupplInfo9.htm.

\section{References}

1. Parker, H.G., Kim, L.V., Sutter, N.B. et al. (2004), 'Genetic structure of the purebred domestic dog', Science Vol. 304, pp. 1160-1164.

2. Vilà, C., Savolainen, P., Maldonado, J.E. et al. (1997), 'Multiple and ancient origins of the domestic dog', Science Vol. 276, pp. 1687-1689.

3. Savolainen, P., Zhang, Y.P., Luo, J. et al. (2002), 'Genetic evidence for an East Asian origin of domestic dogs', Science Vol. 298, pp. $1610-1613$.

4. Irion, D., Schaffer, A.L., Famula, T.R. et al. (2003), 'Analysis of genetic variation in $28 \mathrm{dog}$ breed populations with 100 microsatellite markers', J. Hered. Vol. 94, pp. 81-87.

5. Bunney, W.E., Bunney, B.G., Vawter, M.P. et al. (2003), 'Microarray technology: A review of new strategies to discover candidate vulnerability genes in psychiatric disorders', Am. J. Psychiatry Vol. 160, pp. 657-666.

6. Nevins, J.R., Huang, E.S., Dressman, H. et al. (2003), 'Towards integrated clinico-genomic models for personalized medicine: Combining gene expression signatures and clinical factors in breast cancer outcomes prediction', Hum. Mol. Genet. Vol. 12, pp. R153-R157.

7. Gibson, G. (2003), 'Population genomics: Celebrating individual expression', Heredity Vol. 90, pp. 1-2.
8. Wolfinger, R.D., Gibson, G., Wolfinger, E.D. et al. (2001), 'Assessing gene significance from cDNA microarray expression data via mixed models', J. Comput. Biol. Vol. 8, pp. 625-637.

9. Kerr, M.K., Martin, M. and Churchill, G. (2000), 'Analysis of variance for gene expression microarray data', J. Comput. Biol. Vol. 7, pp. 819-837.

10. Efron, B. and Tibshirani, R. (2002), 'Empirical Bayes methods and false discovery rates for microarrays', Genet. Epidemiol. Vol. 23, pp. 70-86.

11. Townsend, J.P. and Hartl, D.L. (2002), 'Bayesian analysis of gene expression levels: Statistical quantification of relative mRNA level across multiple strains or treatments', Genome Biol. Vol. 3, Research0071.

12. Jin, W., Riley, R., Wolfinger, R.D. et al. (2001), 'The contributions of sex, genotype and age to transcriptional variance in Drosophila melanogaster', Nat. Genet. Vol. 29, pp. 389-395.

13. Cheung, V.G. and Spielman, R.S. (2002), 'The genetics of variation in gene expression', Nat. Genet. Vol. 32 (Suppl.), pp. 522-525.

14. Vawter, M.P., Evans, S., Choudary, P. et al. (2003), 'Gender-specific gene expression in post-mortem human brain: Localization to sex chromosomes', Neuropsychopharmacology Vol. 29, pp. 373-384.

15. Enard, W., Khaitorich, P., Klose, J. et al. (2002), 'Intra- and interspecific variation in primate gene expression patterns', Science Vol. 296, pp. $340-343$.

16. Hsieh, W.P., Chu, M., Wolfinger, R.D. and Gibson, G. (2003), 'Mixed model reanalysis of primate data reveals species and tissue biases in gene expression', Genetics Vol. 165, pp. 747-757.

17. Khaitovich, P., Muetzel, B., She, X. et al. (2004), 'Regional patterns of gene expression in human and chimpanzee brains', Genome Res. Vol. 14, pp. $1462-1473$.

18. Sandberg, R., Yasuda, R., Pankratz, D.G. et al. (2000), 'Regional and strain-specific gene expression mapping in the adult mouse brain', Proc. Natl. Acad. Sci. USA Vol. 97, pp. 11038-11043.

19. Pavlidis, P. and Noble, W.S. (2001), 'Analysis of strain and regional variation in gene expression in mouse brain', Genome Biol. Vol. 2, Research0042.

20. Saetre, P., Lindberg, J., Leonard, J.A. et al. (2004), 'From wild wolf to domestic dog: Gene expression changes in the brain', Mol. Brain Res. Vol. 126, pp. $198-206$.

21. Roberts, M.C., Hitte, C.C., Hendrickson, J.A. et al. (2003), 'Characterization and radiation hybrid mapping of expressed sequence tags from the canine brain', Mamm. Genome Vol. 14, pp. 203-213.

22. Higgins, M.A., Berridge, B.R., Mills, B.J. et al. (2003), 'Gene expression analysis of the acute phase response using a canine microarray', Toxicol. Sci. Vol. 74 , pp. $470-484$.

23. Zubakov, D., Hoheisel, J.D., Kluxen, F.W. et al. (2003), 'Late ischemic preconditioning of the myocardium alters the expression of genes involved in inflammatory response', FEBS Lett. Vol. 547, pp. 51-55.

24. Asakura, M., Takashima, S. et al. (2003), 'Canine DNA array as a potential tool for combining physiology and molecular biology', Circ. J. Vol. 67 , pp. $788-792$.

25. Balkovetz, D.F., Gerrard, Jr, E.R., Li, S. et al. (2004), 'Gene expression alterations during HGF-induced dedifferentiation of a renal tubular epithelial cell line (MDCK) using a novel canine DNA microarray', $A m$. J. Physiol. Renal Physiol. Vol. 286, pp. F702-F710.

26. Eberwine, J. (1996), 'Amplification of mRNA populations using aRNA generated from immobilized oligo(dT)-T7 primed cDNA', Biotechniques Vol. 20, pp. 584-591.

27. Eisen, M.B., Spellman, P.T., Brown, P.O. and Botstein, D. (1998), 'Cluster analysis and display of genome-wide expression patterns', Proc. Natl. Acad. Sci. USA Vol. 95, pp. 14863-14868.

28. Gibson, G. and Wolfinger, R.D. (2004), 'Gene expression profiling with the SAS Microarray Solution', In Saxton, A.M., (ed), Genetic Analysis of Complex Traits with SAS, Users Press/SAS Publishing, Cary, NC, Chapter 11, (in press).

29. Su, A.I., Cooke, M.P., Ching, K.A. et al. (2002), 'Large-scale analysis of the human and mouse transcriptomes', Proc. Natl. Acad. Sci. USA, Vol. 99, pp. $4465-4470$.

30. Lynch, M. and Force, A. (2000), 'The probability of duplicate gene preservation by subfunctionalization', Genetics Vol. 154, pp. 459-473. 
31. Ryan, M.M., Huffaker, S.J., Webster, M.J. et al. (2004), 'Application and optimization of microarray technologies for human postmortem brain studies', Biol. Psychiatry Vol. 55, pp. 329-336.

32. Meiklejohn, C.D., Parsch, J., Ranz, J.M. and Hartl, D.L. (2003), 'Rapid evolution of male-biased gene expression in Drosophila', Proc. Natl. Acad. Sci. USA Vol. 100, pp. 9894-9899.

33. Rifkin, S.A., Kim, Y. and White, K.P. (2003), 'Evolution of gene expression in the Drosophila melanogaster subgroup', Nat. Genet. Vol. 33, pp. $138-144$.
34. Oleksiak, M.F., Churchill, G.A. and Crawford, D.L. (2002), 'Variation in gene expression within and among natural populations', Nat. Genet. Vol. 32, pp. 261-266.

35. Sabol, S.Z., Hu, S. and Hamer, D. (1998), 'A functional polymorphism in the monoamine oxidase A gene promoter', Hum. Genet. Vol. 103, pp. $273-279$.

36. Lesch, K.P., Bengel, D., Heils, A. et al. (1996), 'Association of anxietyrelated traits with a polymorphism in the serotonin transporter gene regulatory region', Science Vol. 274, pp. 1527-1530. 